\title{
ARTICLE
}

\section{Twofold advantage of gas bubbling for the advanced solvothermal preparation of efficient YAG:Ce nanophosphors}

Received 00th January 20xx, Accepted 00th January 20xx DOI: $10.1039 / x 0 \times x 00000 x$

\begin{abstract}
Alexandra Cantarano, ${ }^{a}$ Denis Testemale, ${ }^{a}$ Sonia Sousa Nobre, ${ }^{\mathrm{b}}$ Audrey Potdevin, ${ }^{\mathrm{c}}$ Rémy Bruyère, ${ }^{\mathrm{a}}$ Aude Barbara, ${ }^{a}$ Jean-Louis Hazemann, ${ }^{a}$ Alain Ibanez, ${ }^{a}$ Géraldine Dantell ${ }^{{ }^{*}}$

With the goal of controlling the oxidation state of cerium ions in Ce-doped $\mathrm{Y}_{3} \mathrm{Al}_{5} \mathrm{O}_{12}$ nanocrystals (YAG:Ce), different gases $\left(\mathrm{Ar} / \mathrm{H}_{2}, \mathrm{O}_{2}, \mathrm{Ar}\right)$ were bubbled inside the solution of precursors and solvent prior to the solvothermal synthesis. As assessed by X-ray absorption spectroscopy, this protocol modifies the $\mathrm{Ce}^{3+}: \mathrm{Ce}^{4+}$ ratio in YAG:Ce nanocrystals, although it does not allow to prevent completely the $\mathrm{Ce}^{3+} \rightarrow \mathrm{Ce}^{4+}$ oxidation. In addition, gas bubbling strongly influences the size and crystal quality of YAG:Ce nanocrystals, as evidenced by X-ray diffraction and Transmission Electron Microscopy. Indeed, the presence of bubbles in the precursor solution induces heterogeneous nucleation, leading to an earlier nucleation step. This favors the growth and the high crystallinity of YAG:Ce nanocrystals leading to record iQY at around $52 \%$ for $60 \mathrm{~nm}$ sized nanocrystals and $66 \%$ for those of $200 \mathrm{~nm}$.
\end{abstract}

\section{Introduction}

With the advent of the semiconductor-based nanotechnologies, miniaturization has become more and more accessible, leading to tremendous innovations in electronics, photonics, sensors, etc. Over the last decade, solid-state lighting, through LightEmitting Diodes (LEDs) and more recently with Organic LED technologies, has emerged, offering in particular drastic energy savings. However, many challenges remain in this field, such as the improvement of light extraction from white LEDs or displays, for greater efficiency. Recent developments include elaboration of micro-LEDs and semiconductor nanostructuration. ${ }^{1,2}$ Phosphor elaboration is also a key factor in optimizing the efficiency and stability of phosphor-converted LED (pc-LED) lighting devices.3,4,5,6 In this context and for the development of new applications, particularly for miniaturization involving solution-based deposition techniques (e.g. ink-jet printing 7 ), it is required to couple the semiconducting heterostructures with phosphors of reduced size. ${ }^{8}$ Some pioneer works have been performed using submicronic $\mathrm{Ce}^{3+}$-doped $\mathrm{Y}_{3} \mathrm{Al}_{5} \mathrm{O}_{12}$ (YAG:Ce) phosphors, the currently used phosphor in white light LEDs. ${ }^{9,10}$ To meet the phosphor size demand $(<100 \mathrm{~nm})$, there is a great interest in mastering the chemical synthesis of YAG:Ce nanocrystals (NCs), including the control of their size and crystal quality to ensure high photoluminescence efficiency, quantified by the internal quantum yield ( $i Q Y$ ). Among the different methods proposed to synthesize YAG:Ce NCs, the prominent ones comprise combustion, ${ }^{11,12}$ coprecipitation, ${ }^{13,14}$ sol-gel, ${ }^{15,16,17}$ and

\footnotetext{
a. Univ. Grenoble Alpes, CNRS, Grenoble INP, Institut Néel, 38000 Grenoble, France.

b. Univ. Grenoble Alpes, CEA, LITEN DTNM, F-38054 Grenoble, France

c. Université Clermont Auvergne, CNRS, SIGMA Clermont, Institut Pascal, F-63000

Clermont-Ferrand, France.
}

solvothermal processes. ${ }^{18,19,20}$ In most of these techniques, an annealing step is required to crystallize YAG phase, inducing NC agglomeration if no care is taken during the thermal treatment. A recent paper reports the precipitation and crystallization of NCs previously embedded inside a $\mathrm{LiCl}$ matrix, preventing their aggregation, resulting in YAG:Ce NCs with a size smaller than $100 \mathrm{~nm}$ and a $i Q Y$ of $50 \%$. However, the presence of $\mathrm{CeO}_{2}$ parasitic phase is also detected. ${ }^{21}$ Finally, the solvothermal method, often employed to produce nanocrystals with good crystallinity, ${ }^{22}$ enables to produce non-aggregated nanometersized YAG:Ce NCs that can be then be dispersed in ethanol thanks to the presence of organic groups resulting from the synthesis solvent and forming a stabilizing corona around the NCs that can thus be used individually. ${ }^{23,24,25}$ However, two challenges remain for the solvothermal method: (1) to control the nanoparticle crystal quality and (2) to master the cerium oxidation state.

Regarding the first point, the control of crystal quality implies to master nucleation and growth mechanisms of YAG:Ce NCs. The nucleation step is particularly difficult to control in autoclave, which can be considered as a "black box". Indeed, the pressure is generally autogenous and thus increases gradually as well as temperature, leading to non-stabilized and inappropriate conditions as nucleation must be confined in time, or eventually in space. ${ }^{26}$ This generally leads, for YAG NC syntheses, to a "cauliflower" morphology, with nanoparticles being formed of many small NCs (a few $\mathrm{nm}$ ), which is certainly the consequence of an uncontrolled nucleation step. ${ }^{27,28}$ As a result, due to numerous surface defects and a lack of good crystallinity of these particles, their photoluminescence efficiency is limited with weak $i Q Y=$ ca. $20-30 \% .^{29,30,31}$ Strategies to increase the $i Q Y$, while preserving the nanometer size of particles, include surface modification (PEG coating, ${ }^{32}$ growth of a protective layer, ${ }^{33}$ surface passivation ${ }^{34}$ ) and protective thermal 
annealing. ${ }^{35}$ The surface-modified NCs present $i Q Y$ around 35 $\%$, while the latter method leads to NCs with $i Q Y$ of $60 \%$, but this multi-step process cannot be scaled up due to a timeconsuming and hazardous process involving hydrofluoric acid..$^{35}$ The second challenge concerns the Ce oxidation state control. We recently showed, through in situ X-Ray absorption spectroscopy (X-ray Absorption Near Edge Spectroscopy, XANES), that the solvothermal method induces the partial oxidation of $\mathrm{Ce}^{3+}$ ions into $\mathrm{Ce}^{4+}$ ions upon synthesis. ${ }^{36}$ Not only $\mathrm{Ce}^{4+}$ ions are known to be optically silent (i.e. do not participate to photoluminescence), ${ }^{37,38}$ but they also may have a role as luminescent quenchers. ${ }^{39}$ One hypothesis is that they would act as traps for $\mathrm{Ce}^{3+}$ luminescence, thus reducing the $i Q Y .{ }^{40}$

In this study, we initially tackled the second issue, with the aim of preserving cerium ions in their $3+$ oxidation state by bubbling a reducing gas into the precursor solutions. The idea was to mimic the solid-state reaction for the formation of YAG:Ce ${ }^{3+}$ phosphors, for which the annealing treatment is performed under $\mathrm{Ar} / \mathrm{H}_{2}$ gas to maintain cerium ions in their $3+$ oxidation state. ${ }^{41}$ The relative proportion $\mathrm{Ce}^{3+}: \mathrm{Ce}^{4+}$ was monitored by XANES spectroscopy. In addition, we evidenced that gas bubbling has a significant effect on the nucleation and growth steps of NCs, allowing the production of high quality, nonaggregated YAG:Ce NCs through a one-step process exhibiting one of the highest $i Q Y S$ ever reported in the literature.

\section{Results and Discussion}

In order to lower the $\mathrm{Ce}^{3+}$ oxidation during the synthesis of YAG:Ce nanocrystals, a reducing gas $\left(\mathrm{Ar} / \mathrm{H}_{2}\right)$ was bubbled into the initial solution of 1,4-butanediol and precursors for two hours at low flow rate, prior to the solvothermal reaction. For comparison, another YAG:Ce sample was synthesized after bubbling an oxidizing gas $\left(\mathrm{O}_{2}\right)$, into the initial solution during 2 h. Finally, a third sample was prepared without gas bubbling. These samples were characterized by XANES and photoluminescence spectroscopy to examine their $\mathrm{Ce}^{3+}: \mathrm{Ce}^{4+}$ ratio and respective $i Q Y$. Following these results, the bubbling process was optimized and its impact on the NC structure and morphology was investigated.

Controlling the $\mathrm{Ce}^{3+}: \mathrm{Ce}^{4+}$ ratio by gas bubbling. XANES spectra of both nanophosphor samples involving gas bubbling are shown in Figure 1, along with XANES spectra of reference samples. Note that the staring cerium precursor is $\mathrm{Ce}(\text { acetate })_{3}$ and contains only $\mathrm{Ce}^{3+}$ ions (Figure S1). ${ }^{42}$ Both nano-YAG powders contain $\mathrm{Ce}^{4+}$ ions, as evidenced by the presence of the peak at $5739 \mathrm{eV} .{ }^{43,44}$ However, the proportion of $\mathrm{Ce}^{4+}$ is slightly higher in the sample resulting from the $\mathrm{O}_{2}$ bubbling $\left(\mathrm{Ce}^{3+}: \mathrm{Ce}^{4+}=\right.$ $64: 36 \pm 2$ ) than in the sample resulting from the $\mathrm{Ar} / \mathrm{H}_{2}$ bubbling $\left(\mathrm{Ce}^{3+}: \mathrm{Ce}^{4+}=72: 28 \pm 2\right)$. This trend is consistent with the reducing character of the $\mathrm{Ar} / \mathrm{H}_{2}$ gas and the oxidizing character of $\mathrm{O}_{2}$ gas. However, on the contrary to the solid-gas reaction occurring in the solid-state process, the presence of $\mathrm{H}_{2}$ dissolved in the solvent does not enable to prevent significantly the $\mathrm{Ce}^{3+} \rightarrow \mathrm{Ce}^{4+}$ oxidation reaction.

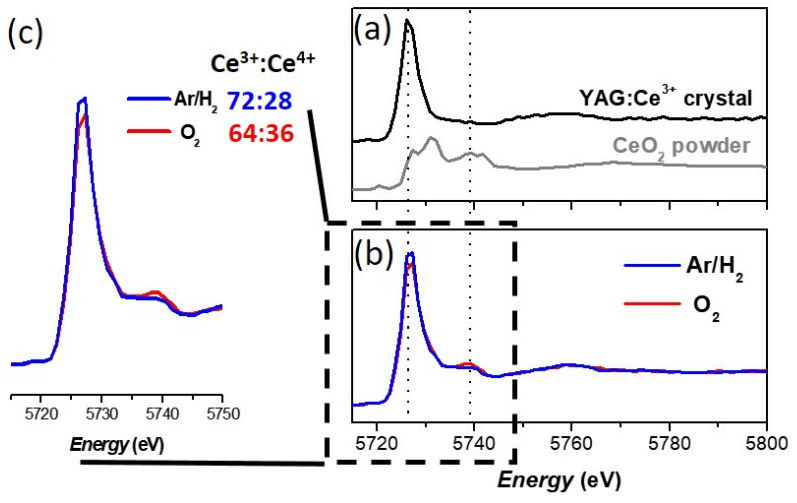

Figure 1. (a) XANES spectra of the standards used for the linear combination (b) XANES spectra of the YAG:Ce nanophosphors obtained by solvothermal synthesis performed after $\mathrm{Ar} / \mathrm{H}_{2}$ or $\mathrm{O}_{2}$ gas bubbling for two hours in the precursor solution. (c) Zoom on the peaks characteristics of $\mathrm{Ce}^{3+}$ absorption (at $5725 \mathrm{eV}$ ) and of $\mathrm{Ce}^{4+}$ absorption (at $5738 \mathrm{eV}$ ). The $\mathrm{Ce}^{3+}: \mathrm{Ce}^{4+}$ ratio is indicated for each sample.

\begin{tabular}{|l|c|c|}
\hline Sample & $\mathrm{Ce}^{3+}: \mathrm{Ce}^{4+}$ ratio $( \pm 2)$ & $i Q Y( \pm 5 \%)$ \\
\hline $\mathrm{O}_{2}$ bubbling & $64: 36$ & $46 \%$ \\
\hline $\mathrm{Ar} / \mathrm{H}_{2}$ bubbling & $72: 28$ & $45 \%$ \\
\hline No bubbling & $68: 32$ & $38 \%$ \\
\hline \multicolumn{2}{|c|}{ Table $1 . \mathrm{Ce}^{3+} \cdot \mathrm{Ce}^{4+}$ ratios for YAG:C NCs obtained after bubbling $\mathrm{O}_{2}$ or $\mathrm{Ar} / \mathrm{H}_{2}$ gas }
\end{tabular}

Table 1. $\mathrm{Ce}^{3+}: \mathrm{Ce}^{4+}$ ratios for YAG:Ce NCs obtained after bubbling $\mathrm{O}_{2}$ or $\mathrm{Ar} / \mathrm{H}_{2}$ gas for two hours in the precursor solution, or without any bubbling. Their associated $i Q Y$ values are also reported

Interestingly, both samples arising from $\mathrm{Ar} / \mathrm{H}_{2}$ and $\mathrm{O}_{2}$ bubbling, although they have slightly different $\mathrm{Ce}^{3+}: \mathrm{Ce}^{4+}$ ratios, present the same high $i Q Y$ value (around $45 \%$, Table 1). This would suggest that showing that $\mathrm{Ce}^{4+}$ ions do not act as traps for $\mathrm{Ce}^{3+}$ luminescence. On the other hand, the nanophosphor obtained without gas bubbling exhibits a significantly lower iQY (38\%), while the $\mathrm{Ce}^{3+}: \mathrm{Ce}^{4+}$ ratio is in between the values of the two bubbled samples $\left(\mathrm{Ce}^{3+}: \mathrm{Ce}^{4+}=68: 32 \pm 2\right)$ (Table 1). Hence, the nature of the gas bubbled inside the precursor solution positively influences the proportion of $\mathrm{Ce}^{3+}: \mathrm{Ce}^{4+}$ in YAG:Ce NCs but does not allow to prevent completely $\mathrm{Ce}^{3+}$ oxidation into $\mathrm{Ce}^{4+}$, which is mainly driven by the solvothermal process itself. Indeed, the cerium oxidation most likely comes from the partial decomposition of 1,4-butanediol into tetrahydrofuran and water starting from $250^{\circ} \mathrm{C}$ and 180 bar during synthesis, 25,45 and from the partial decomposition of the organics precursors when working at high pressure, just above $300^{\circ} \mathrm{C} .46,47,48$ Interestingly, the presence of gas bubble influences the $i Q Y$ of YAG:Ce NCs as an $i Q Y$ of $38 \%$ is obtained when no bubbling is performed, whereas an $i Q Y$ value of $45 \%$ is obtained when a gas is bubbled in the precursor solution.

\section{Influence of gas bubbling on YAG:Ce NC morphology}

Hereafter, we present in detail how the bubbling treatment impacts the YAG:Ce NC morphology and optical properties. Different YAG:Ce NC samples were obtained, resulting from a synthesis in 1,4-butanediol or in 1,5-pentanediol. The latter has been chosen for its higher viscosity (128 mPa.s for 1,5pentanediol ${ }^{49}$ vs $84.9 \mathrm{mPa} . \mathrm{s}$ for 1,4 -butanediol, 50 at $20^{\circ} \mathrm{C}$ ), allowing a more efficient bubbling in the precursor solutions, and potentially a more efficient bubble trapping. 

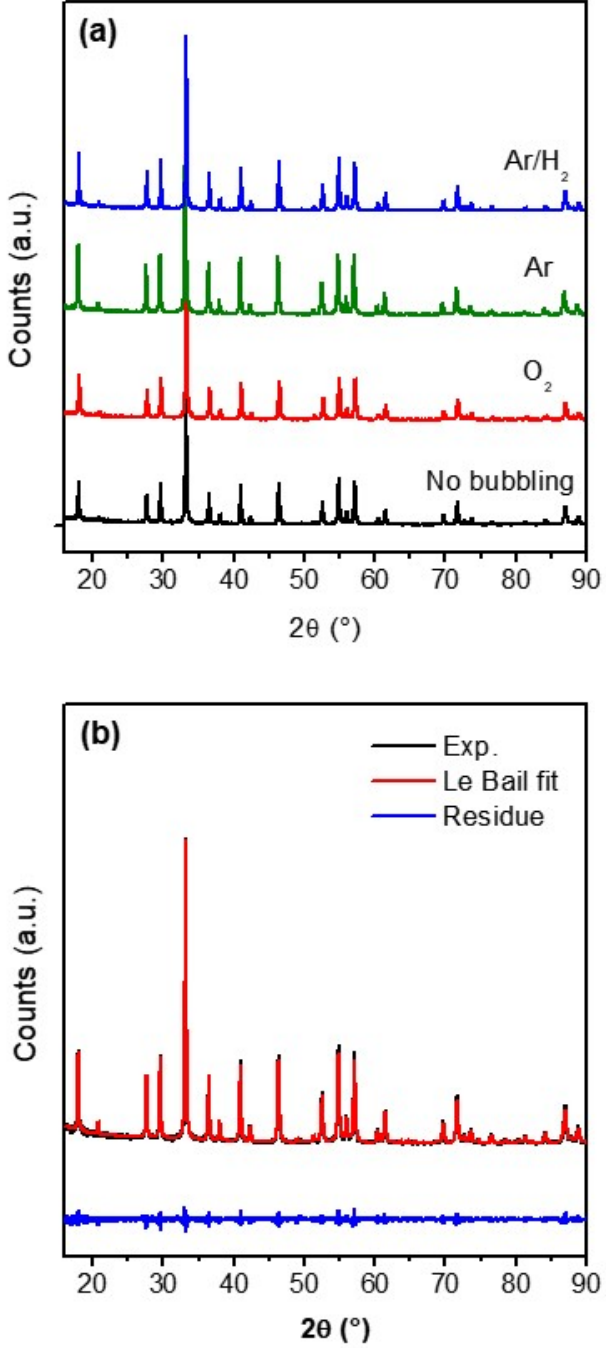

Figure 2: (a) PXRD patterns of YAG:Ce nanophosphors resulting from the bubbling of different gas for 4 hours in 1,4-butanediol. For comparison, the PXRD pattern of the sample obtained without bubbling is reported (b) PXRD pattern of YAG:Ce nanophosphors obtained without bubbling and its associated Le Bail fit.

Different $\mathrm{Ar} / \mathrm{H}_{2}$ bubbling durations (0, 2, 4 and $24 \mathrm{~h}$ ) were studied, with identical solvothermal conditions $\left(320^{\circ} \mathrm{C}, 200\right.$ bar). The optimal bubbling duration has been adjusted to $4 \mathrm{~h}$ based on NC coherence length evolution (Figure S2). As a reference, their morphological and optical properties will be compared to a sample obtained without bubbling.

Pure garnet-type YAG phase was successfully synthesized for all samples, as shown in Figure 2a from powder X-ray Diffraction (PXRD) pattern analysis. Figure $\mathbf{2 b}$ presents a typical PXRD pattern recorded for the sample prepared without bubbling and its associated Le Bail fit. From Le Bail fits, the unit cell parameter, $a$, was determined, along with the coherence length. Only PXRD patterns of the samples synthesized in 1,4butanediol are given as typical examples in Figure $\mathbf{2}$.

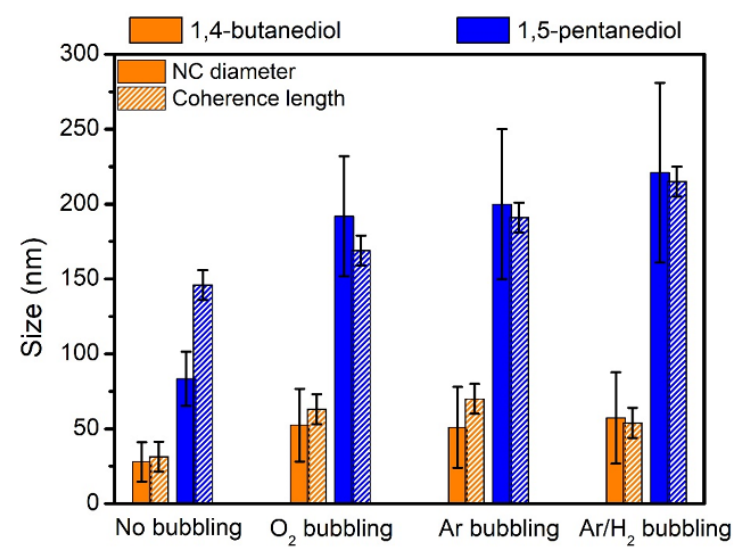

Figure 3: Diameter (filled) and Lc (patterned) of YAG:Ce NCs synthesized in 1,4butanediol (orange) and 1,5-pentanediol (blue) with no bubbling and with $4 \mathrm{hr}$ bubbling of $\mathrm{O}_{2}, \mathrm{Ar}$ and $\mathrm{Ar} / \mathrm{H}_{2}$ gas.

Similar diffraction patterns were obtained for the samples synthesized in 1,5-pentanediol also confirming the absence of crystallized impurity.

The unit cell parameter was determined to be $a=12.066 \pm$ $0.013 \AA$ for all samples synthesized in 1,4-butanediol and $a=$ $12.090 \pm 0.025 \AA$ for the samples synthesized in $1,5-$ pentanediol. On the other hand, the different bubbling treatments have no impact on the lattice parameter, whatever the nature of gas and bubbling time. These unit cell values, $12.066 \AA$ and $12.090 \AA$, are larger than for the bulk YAG crystal, which is of $12.012 \AA .{ }^{41}$ These higher unit cell parameters for nano-YAG are reported in the literature and decrease with thermal treatments, $35,51,52$ which suggests that it comes from the local disorder of the nanometer sized NCs and not because of the synthesis process itself. It might arise from internal strain inside NCs. ${ }^{36}$ Indeed, maximal internal strains, $\varepsilon$, inside the samples were deduced from the Le Bail fits: $\varepsilon=12 \pm 5 \%$ for all NCs synthesized in 1,4-butanediol and $\varepsilon=26 \pm 6 \%$ for all NCs synthesized in 1,5-pentanediol. The nature of the gas does not seem to impact the strains inside the NCs but the nature of the solvent did. Moreover, a higher maximal strain for the samples synthesized in 1,5-pentanediol is in good agreement with a higher unit cell parameter.

The coherence length, $L_{c}$, of YAG:Ce NCs calculated from PXRD and their diameter obtained from TEM image analysis are compared in Figure 3 for samples synthesized in 1,4-butanediol and in 1,5-pentanediol, respectively. In both series, $L_{c}$ as well as the average size increase when gas bubbling is performed in the initial solutions whatever the nature of the gas. Syntheses made in 1,5-pentanediol result in also higher NC diameter and $L_{c}$ than in 1,4-butanediol.

The $L_{c}$ increase observed in both solvents when bubbling gas can be associated with the parallel increase of the NC diameter, but also with an improved crystallinity in the samples. Hence, bubbling gas in the precursor solutions allows the preparation of YAG:Ce NCs with bigger size and improved crystallinity, whatever the nature of the gas. 

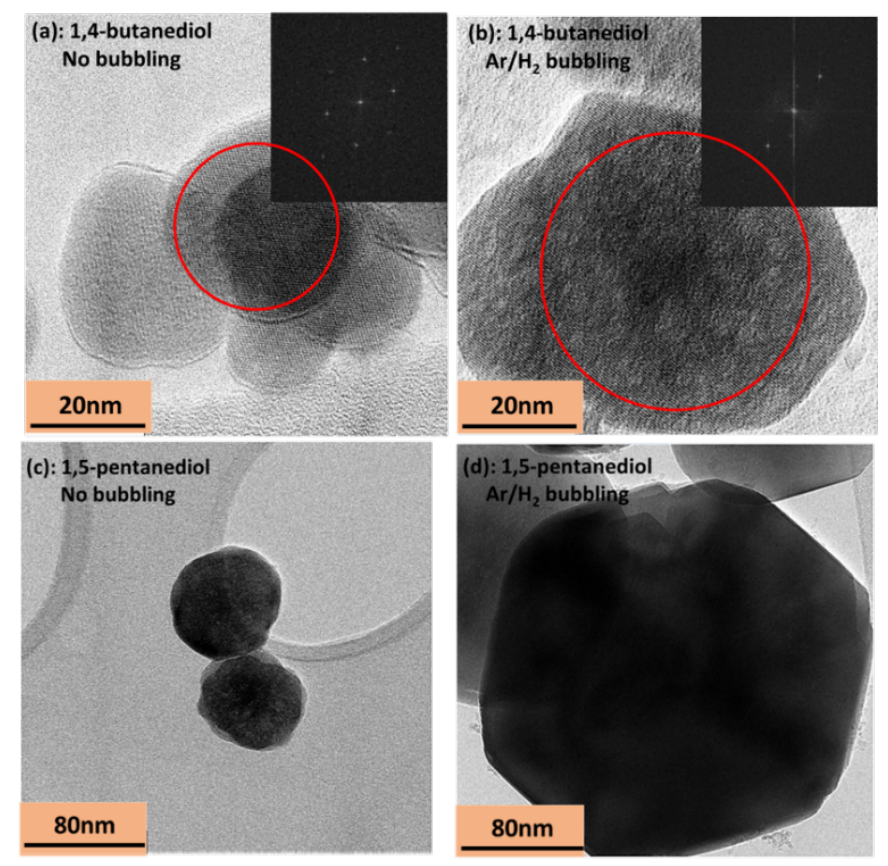

Figure 4: TEM images of YAG:Ce NCS as a function of bubbling treatment and solvent: (a) No bubbling and (b) $\mathrm{Ar} / \mathrm{H}_{2} 4 \mathrm{~h}$ - bubbling in 1,4-butanediol, (c) no and (b) the Fourier transforms corresponding to the areas circled in red are presented, proving the single crystal character of the NCs.

TEM images (Figure 4) show that the NCs obtained in this study present a good crystallinity, even for samples prepared with no bubbling, compared to cauliflower-shape NCs, commonly seen in the literature, which arise from a massive agglomeration of primary YAG:Ce nanoparticles. ${ }^{19,28,32}$ This is due to the synthesis conditions of the modified solvothermal process, where an external pressure of 200 bar is $a b$ initio applied..$^{36}$ The synthesis at $320^{\circ} \mathrm{C}$ and around 200 bar increases the crystallinity of the particles, with the atomic planes running over the single crystals as observed in TEM images (Figure 4 (a) and (b)) and the crystalline facets clearly visible. Moreover, when using the bubbling protocol, TEM pictures show an increase of the NCS size (Figure $\mathbf{4}$ (c) and (d) and Figure $\mathbf{S} \mathbf{3}$ for images taken at lower magnification) as well as their crystallinity (more faceted crystals) in perfect agreement with the coherence lengths extracted from PXRD patterns. A consequence of these morphological changes of NCs is the enhancement of their iQY (Figure 5). Indeed, size increase induces a reduction of NC specific surface, leading to fewer surface traps responsible for non-radiative de-excitations. Consistently, as NCs obtained in 1,5-pentanediol are bigger than those obtained in 1,4butanediol, higher $i Q Y$ s are observed: for the former $50 \%$ with no bubbling and $57 \%$ to $66 \%$ when bubbling, while for the latter we measured an $i Q Y$ of $38 \%$ with no bubbling and an iQY between $42 \%$ and $52 \%$ when bubbling, Figure 5 . Thus, a record iQY of $66 \%$ has been obtained for $200-\mathrm{nm}$ NCs synthesized in 1,5-pentanediol with an Ar-bubbling. In addition, one can note the high $i Q Y$ (52\%) obtained for the 60-nm NCs, whose size is more appropriate for the targeted applications.

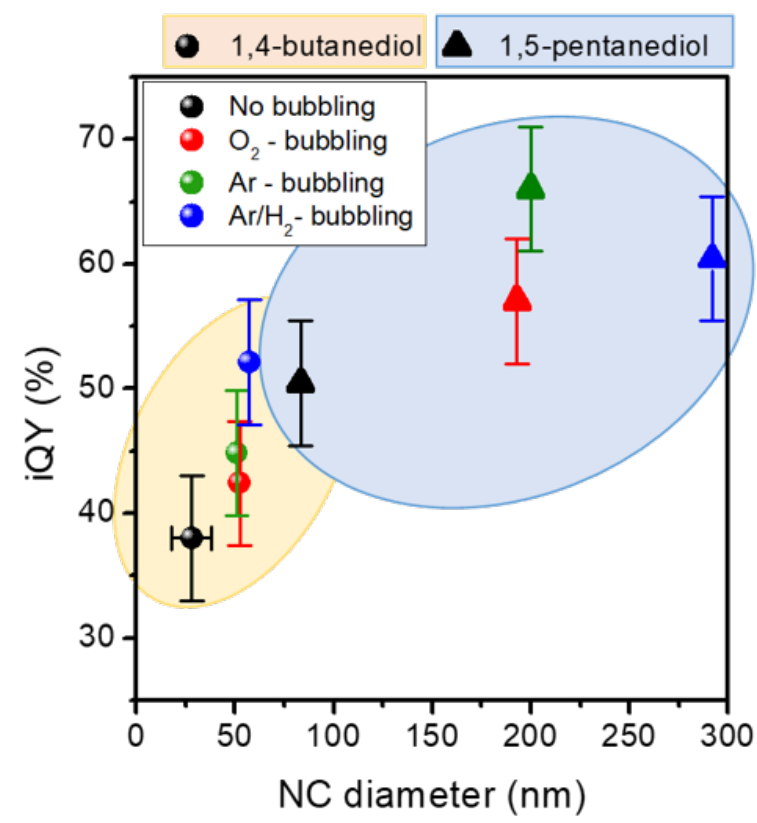

Figure 5: iQY for the different samples synthesized in (a) 1,4-butanediol (circles) and in (b) 1,5-pentanediol (triangles).

Undoubtedly, the bubbling process notably impacts the nanocrystallisation mechanisms during the solvothermal process. The most likely hypothesis is that the presence of bubbles in the precursor solution promotes an earlier nucleation step. This then allows more time to the nuclei to grow and coalesce through the self-oriented coalescence mechanism, previously observed by several works. ${ }^{27,53}$ Thus, a significant improvement in crystallinity is observed by PXRD and TEM: increase of the coherence length, Figure 3, and appearance of NC facets, respectively. This earlier nucleation step compared to no bubbling experiments is certainly favoured by a heterogeneous nucleation of YAG NCs at the bubblesolution interface. ${ }^{54}$ To confirm this hypothesis and, as the presence of gas bubbles under pressure is a delicate and rather undocumented matter, we carried out in situ Dynamic Light scattering (DLS) measurements using a home-made autoclave with optical windows, described in [55] and illustrated in Figure 6a. The experiment was performed with the solvent only after gas $\left(\mathrm{Ar} / \mathrm{H}_{2}\right)$ bubbling in order to avoid any parasitic diffusion that could result from the addition of metal precursors. Gas bubbles were either directly visualized using a 2 MPixel Basler acA1600-20uc camera, based on a Sony CCD sensor with $4 \times 4$ $\mu \mathrm{m}^{2}$ pixel size, or, for smaller bubbles, detected through in situ DLS experiments using a portable Cordouan particle size analyser (VASCO Kin). The pressure was increased progressively up to 100 bar. Under atmospheric pressure, bubbles with a size of $40 \mu \mathrm{m}$ were observed by the camera. As the pressure increases, their diameter decreases $(10 \mu \mathrm{m}$ at 5 bar) due to the compression and the increased gas solubility. ${ }^{56}$ At 100 bar, the camera was unable to observe any microscopic bubbles, indicating either the disappearance of bubbles at such pressure, or the presence of nanometer-sized bubbles. 
(a)

(c)

\section{Without bubbling}

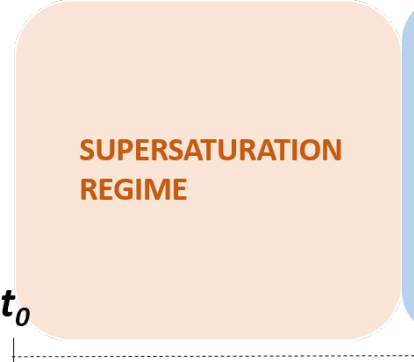

\section{NUCLEATION}

(b)

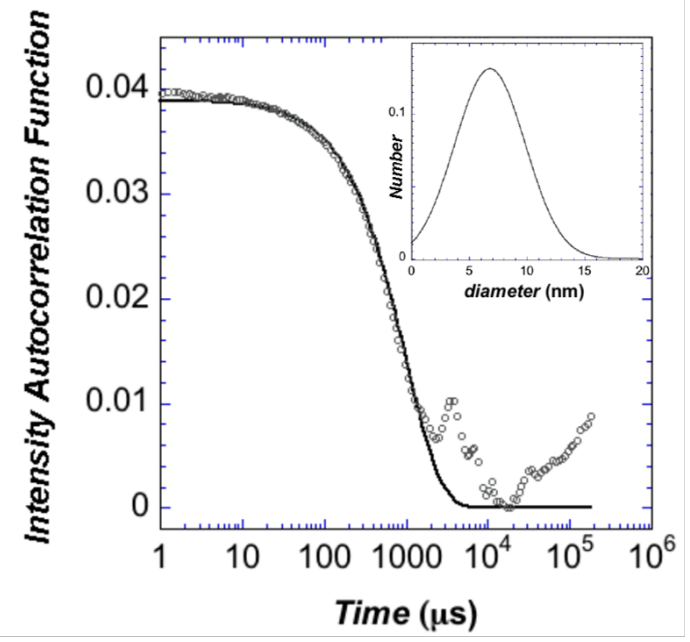

\section{With bubbling}

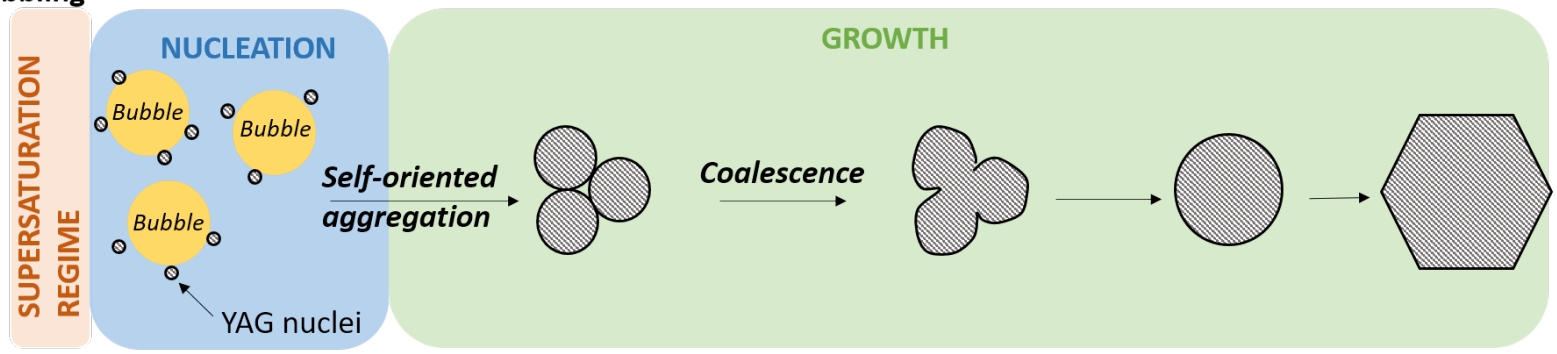

Figure 6: (a) Scheme of the experimental set-up used to assess the presence of gas bubbles under pressure. (b) Experimental (diamonds) and calculated (line) IACF measured in the solvent after gas bubbling, under 100 bar. Inset: diameter distribution of the bubbles obtained from the parameters of the calculated IACF. (c) Scheme
of the proposed explanation for increased growth and crystallinity when bubbling a gas inside the precursor solution (bottom) compared to with no bubbling (top) as a function of the synthesis time.

At such sizes, the buoyancy of the bubbles is very weak and their motion is consequently dominated by the thermal Brownian movement. Therefore, in situ DLS experiments can be performed to highlight their potential existence. Indeed, as for DLS measurements on colloidal solutions were the particles are driven by a Brownian motion, the movement of the bubbles will induce temporal fluctuations in the intensities of the light they scatter. These fluctuations may be analysed by deriving the normalized autocorrelation functions of the measured intensities (IACF). In the absence of bubbles, the IACF is expected to be flat and equal to 1 during the whole acquisition time. On the contrary, in the presence of diffusing bubbles, the IACF is expected to present an exponential decay as a function of time with a characteristic time related to the size of the nanobubbles. ${ }^{57,58}$ The experiments were realized by illuminating the solution through the optical window and collecting the light scattered at $170^{\circ}$ from the incident beam, through the same window. The recorded IACF is presented in Figure $\mathbf{6 b}$ and clearly shows the expected exponential time-decay that signs the presence of bubbles. However, because of the weakness of the scattered intensities, due to the small size of the bubbles, the amplitude of the ACF is weak and a large background signal is observed. A fitting of the IACF was performed following the well-known cumulants method ${ }^{59}$ to access the values of the mean time-decay $\tau_{c}$ and that of the polydispersity index (PDI), which is related to the width of the diameter distribution. The bubble diameter is then calculated knowing that $\tau_{c}=1 / q^{2} D$, where $|q|=4 \pi n \sin (\theta / 2) / \lambda$ is the scattering vector, $n$ being the solvent refractive index, $\theta$ the scattering angle and $\lambda$ the laser wavelength; and $D=\left(k_{B} T / \eta\right) / 3 \pi d$ is the translational diffusion coefficient of the bubbles, $k_{B}$ being the Boltzmann constant, $T$ 
and $\eta$ the temperature and the viscosity of the solvent respectively, and $d$ the hydrodynamic diameter of the bubbles. The best fit of the IACF, also presented in Figure $\mathbf{6 b}$, was found for $\tau_{c}=2.2 \pm 0.3 \mathrm{~ms}$, which leads to a mean diameter $d=6 \pm 1$ $\mathrm{nm}$, as $T=20^{\circ} \mathrm{C}, n=1.44, \lambda=635 \mathrm{~nm}$, and $\eta=128 \mathrm{mPa}$.s. The inset of Figure $6 \mathrm{~b}$ represents the diameter distribution obtained considering a normal distribution and a standard deviation of 45 $\%$ providing from the PDI value. It is to note that in situ DLS experiments on small bubbles may lead to a partial heterodyning of the measurements arising from stray light and scattering from the solvent.60 The occurrence of such phenomena may not be completely ruled out here, however, a partial heterodyning would lead to an underestimate of the bubble size of several tens of percent, but does not question their existence.

Bubble-assisted nucleation process has already been demonstrated for coprecipitation reactions, occurring at mild temperature and ambient pressure, ${ }^{61,62}$ but also for sonocrystallisation processes. ${ }^{63,64}$ Here, we demonstrated that under solvothermal conditions, bubbles also modify the nanocrystallisation mechanism, through an heterogeneous nucleation with a significant reduction of the energy barrier for nucleation, compared to homogeneous nucleation. This favoured the occurrence of a strong confinement in time of the nucleation step at the early stage of the solvothermal process (Figure 6c), giving then the time to YAG primary grains to grow and aggregate to form larger NCs by self-oriented coalescence, 27 leading to larger YAG:Ce NCs with enhanced crystal quality (Figure 4) and associated $i Q Y$.

\section{Experimental}

Synthesis of YAG:Ce nanocrystals. A modified-solvothermal process was developed to synthesize $1 \mathrm{~mol} \% \mathrm{Ce}^{3+}$-doped YAG NCs. Compared to conventional solvothermal process where the pressure inside the autoclave is autogenous, an external pressure is here applied, as detailed hereafter. Yttrium acetate tetrahydrate (Alfa Aesar, 99.9\%), cerium acetate dihydrate (STREM chemicals, 99.9\%) and aluminum isopropoxide (Acros organics, $98 \%$ ) with a concentration of $0.18 \mathrm{~mol}^{-\mathrm{L}^{-1}}$ were mixed in stoichiometric proportions and dispersed either in 1,4butanediol or in 1,5-pentanediol. The resulting precursor solutions were stirred at room temperature for $72 \mathrm{~h}$. Then, different gases (oxidizing, $\mathrm{O}_{2}$; neutral; $\mathrm{Ar}$ and reducing; $\mathrm{Ar} / \mathrm{H}_{2}$ gas mixture (95-5 vol.\%)) were bubbled inside the precursor solutions with a volumetric flow rate assessed at $2.5 \mathrm{~cm}^{3} / \mathrm{min} \pm$ $0.5 \mathrm{~cm}^{3} / \mathrm{min}$. Different bubbling duration were studied, from 0 to $24 \mathrm{~h}$. After the bubbling treatment, the precursor solution was poured into a $500 \mathrm{~mL}$ home-made autoclave. Note that one solution without bubbling treatment was synthesized as a reference to study the effect of these treatments on the morphology and optical properties of YAG:Ce NCs. The autoclave was purged and then filled with Argon gas up to 60 bar. The autoclave was heated to $320^{\circ} \mathrm{C}$ for $2 \mathrm{~h} 30$ and then cooled down to room temperature. The pressure inside the autoclave increased from 60 bar at the beginning of the reaction to around 200 bar at the end of the heating step due to the precursor degradation and the liquid to gas transformation of the solvent. The products of the reaction were then washed three times in ethanol through successive centrifugations. For powder characterizations, the products were dried in oven at $100^{\circ} \mathrm{C}$ for $1 \mathrm{~h}$ under air.

Structural characterizations. Powder X-ray diffraction (PXRD) diagrams were recorded on a Siemens D8 Advance diffractometer $\left(\lambda_{\mathrm{Cu}}=1.54056 \AA\right.$ ) . Scans were collected in the $2 \theta$ range $16-90^{\circ}$, with a step of $0.005^{\circ}$ and 1 s of acquisition time. The nanopowders were homogeneously deposited onto a silicon wafer. The unit cell parameter and the coherence length were determined using the Le Bail method on the XRD patterns. ${ }^{65}$

NC morphology and size were investigated by Transmission Electron Microscopy (TEM, Philips CM300). NC average size was obtained from the TEM image analysis. Photoluminescence iQY was measured on nanophosphor powder, using an integrating sphere (Quantaurus-QY Hamamatsu). More specifically, a small amount of powder was deposited in a quartz cuvette, placed at the bottom of an integrating sphere. The powder was excited at $460 \mathrm{~nm}$ and the emission was collected from 500 to $700 \mathrm{~nm}$. The $i Q Y$ was obtained after measuring a blank sample, consisting in the same empty cuvette. The $i Q Y$ measurements were repeated twice for each sample to ensure good repeatability leading to $i Q Y$ values with an uncertainty of $\pm 5 \%$.

XANES experiments, performed in the high energy resolution fluorescence-detected (HERFD) mode at the $L_{3}$ edge of cerium, were achieved at the FAME-UHD beamline at the European Synchrotron Radiation Facility (ESRF Grenoble). ${ }^{66}$ The photon energy was scanned from $5.68 \mathrm{keV}$ to $5.85 \mathrm{keV}$ using a $\mathrm{Si}(220)$ double-crystal monochromator. A helium chamber was mounted between the autoclave, the crystal analyzer spectrometer and the detector to avoid partial beam absorption by air. The X-ray fluorescence signal was recorded with a five Ge (331) crystal analyzer and a Vortex-Ex detector. The beam size was $300 \times 100 \mu \mathrm{m}^{2}$ (horizontal $\times$ vertical FWHM). The energy calibration was done using the $\mathrm{CeO}_{2}$ spectrum. The nanophosphor powder was compacted between two layers of Kapton tape. The goal of these ex situ experiments was to determine the oxidation state of cerium in nanophosphors synthesized after bubbling of various gases in the precursor solution. The $\mathrm{Ce}^{3+}: \mathrm{Ce}^{4+}$ ratio was determined by fitting the experimental data with a linear combination of XANES spectra of standards, i.e. $\mathrm{CeO}_{2}$ nanopowder as a reference for $\mathrm{Ce}^{4+}$ and $\mathrm{Ce}^{3+}$-doped YAG single crystal as a reference for $\mathrm{Ce}^{3+}$, using the Demeter software. ${ }^{67}$ The ratio is given with an uncertainty of $2 \%$.

\section{Conclusions}


Nanocrystals of YAG:Ce were synthesized by a modified solvothermal process involving the bubbling of a gas in the precursor solutions. The set of gas bubbled investigated in this study was selected to contain: one reductive gas, one oxidant and one neutral. First, our study shows that gas bubbling positively influences the $\mathrm{Ce}^{3+}: \mathrm{Ce}^{4+}$ proportion in YAG nanocrystals. Second, the bubbling of any of these gasses favoured the nanocrystallisation through a heterogeneous nucleation mechanism at the early stage of the solvothermal process thanks to the formation of nanometer-sized bubbles acting as nucleation centers. This favoured in a second step the growth of NCs increasing their size and crystallinity. The enhancement in the crystallinity provided a breakthrough in YAG:Ce nanocrystals by enabling us to reach $i Q Y$ of $52 \%$ for 60 $\mathrm{nm}$ crystals and $66 \%$ for $200 \mathrm{~nm}$ crystals, which is among the highest $i Q Y$ ever obtained for YAG:Ce without any annealing treatment. We believe that this method, involving heterogeneous nucleation, a generic process at the bubblesolution interface, can be applied to other types of nanocrystals and would benefit to the entire community working on the nanocrystal synthesis in solution.

\section{Conflicts of interest}

There are no conflicts to declare.

\section{Acknowledgements}

The authors thank the French ANR for funding this research through the NanophosforLED project (ANR-17-CE09-0035-01).

\section{References}

1 R. Ley, L. Chan, P. Shapturenka, M. Wong, S. Denbaars, M. Gordon, "Strain relaxation of InGaN/GaN multi-quantum well light emitters via nanopatterning" Opt. Express, 2019, 27, 30081

2 Q. Jiao, Z. Chen, Y. Feng, S. Li, S. Jiang, J. Li, Y. Chen, T. Yu, X. Kang, B. Shen, G. Zhang, "The effects of nanocavity and photonic crystal in InGaN/GaN nanorod LED arrays" Nanoscale Research Letters (2016) 11, 340

3 P.F. Smet, A.B. Parmentier, D. Poelman, "Selecting conversion phosphors for white light-emitting diodes" J. electrochem. Soc. 2011, 158, R37

4 N. C. George, K. A. Denault, and R. Seshadri "Phosphors for Solid-State White Lighting" Annu. Rev. Mater. Res., 2013, 43, 481-501.

5 Z. Xia, A. Meijerink "Ce3+-Doped garnet phosphors: composition modification, luminescence properties and applications" Chem. Soc. Rev., 2017, 46, 275

6 G. Li, Y. Tian, Y. Zhao, J. Lin "Recent progress in luminescence tuning of Ce3+ and Eu2+-activated phosphors for pc-WLEDs", Chem. Soc. Rev., 2015, 44, 8688

7 Z. Zhan, J. An, Y. Wei, V. Thai Tran, H. Du "Inkjet-printed optoelectronics" Nanoscale (2017) 9, 965

8 Y. Jiang, S.-Y. Cho, M. Shim "Light-emitting diodes of colloidal quantum dots and nanorod heterostructures for future emissive displays" J. Mater. Chem. C, 2018, 6, 2618
9 T. Schimpke, M. Mandl, I. Stoll, B. Pohl-Klein, D. Bichler, F. Zwaschka, J. Strube-Knyrim, B. Huckenbeck, B. Max, M. Muller, P. Veit, F. Bertram, J. Christen, J. Hartmann, A. Waag, H.-J. Lugauer, M. Strassburg "Phosphor-converted white light from blue-emitting InGaN microrod LEDs" Phys. Status Solidi A, 2016, 213, No. 6, 1577-1584

10 N. Guan, X. Dai, A. Messanvi, H. Zhang, J. Yan, E. Gautier, C. Bougerol, F. H. Julien, C. Durand, J. Eymery, M. Tchernychev "Flexible White Light Emitting Diodes Based on Nitride Nanowires and Nanophosphors", ACS photonics, 2016, 3, 597-603

11 A. L. Costa, L. Esposito, V. Medri, and A. Bellosi "Synthesis of Nd-YAG Material by Citrate-Nitrate Sol-Gel Combustion Route" Adv. Eng. Mater., 2007, 9(4) 307-312

12 D. Haranath, H. Chander, P. Sharma, S. Singh "Enhanced luminescence of Y3Al5O12:Ce3+ nanophosphor for white light-emitting diodes" Appl. Phys. Lett., 2006, 89, 173118.

13 E. Caponetti, M.L. Saladino, D. Chillura Martino, L. Pedone, S. Enzo, S. Russu, M., Bettinelli, A. Speghini, "Luminescence properties of neodymium-doped yttrium aluminium garnet obtained by the co-precipitation method combined with the mechanical process" Solid State Phenom., 2005, 106, 7-16.

14 L. Wang, F. Zhao, M. Zhang, T. Hou, Z. Li, C. Pan, H. Huang "Preparation and photoluminescence properties of YAG:Ce3+ phosphors by a series of amines assisted co-precipitation method J. Alloys Cmpd., 2016, 661, 148-154.

15 S. Murai, M.A. Verschuuren, G. Lozano, G. Pirruccio, A.F. Koenderink, J.G. Rivas "Enhanced absorption and emission of Y3Al5O12:Ce3+ thin layers prepared by epoxide-catalyzed solgel method" Opt. Mater. Express 2012, 2, 1111.

16 M. Veith, S. Mathur, A. Kareiva, M. Jilavi, M. Zimmer, V. Huch "Low temperature synthesis of nanocrystalline Y3Al5012 (YAG) and Ce-doped Y3Al5O12 via different sol-gel methods" J. Mater. Chem. 1999, 9, 3069-3079.

17 A. Potdevin, G. Chadeyron, D. Boyer, B. Caillier, and R. Mahiou "Sol-gel based YAG:Tb3+ or Eu3+ phosphors for application in lighting sources" J. Phys. D. Appl. Phys., 2005, 38(17), 32513260.

18 M. Inoue "Glycothermal synthesis of metal oxides" J. Phys. Condens. Matter, 2004, 16, S1291-S1303

19 R. Kasuya, T. Isobe, and H. Kuma "Glycothermal synthesis and photoluminescence of YAG:Ce3+ nanophosphors" J. Alloys Compd., (2006), 408-412, 820-823.

20 H.J. Byun, W.S. Song, Y.S. Kim, H. Yang "Solvothermally grown Ce3+-doped Y3Al5O12 colloidal nanocrystals: spectral variations and white LED characteristics" J. Phys. D: Appl. Phys. 43 (2010) 195401

21 H. F. Gaiser, A. Kuzmanoski, and C. Feldmann "Y3Al5O12:Ce nanoparticles made by ionic-liquid assisted particle formation and LiCl-matrix-treated crystallization" RSC Adv., 2019, 9, 10195-10200

22 Handbook of Synthetic Methodologies and Protocols of Nanomaterials, Vol.1, Chapter 2 "Solvothermal Synthesis of Nanomaterials", Ed. Y. Yin, World Scientific Press (2020)

23 R. Asakura, T. Isobe, K. Kurokawa, T. Takagi, H. Aizaw, M. Ohkubo "Effects of citric acid additive on photoluminescence properties of YAG:Ce3+ nanoparticles synthesized by glycothermal reaction" J. Lumin. (2007) 127, 416-422

24 B. Dong, J. Wang, J Sun, S. Xu, X. Bai, Z. Jiang, Lei Xia, L. Sun, H. Song "Non-photobleaching YAG:Ce nanoparticles for optical imaging with blue excitation" RSC Advances, 2012, 2, 38973905

25 M. Odziomek, F. Chaput, F. Lerouge, M. Sitarz, S. Parola "Highly luminescent YAG:Ce ultra-small nanocrystals, from stable dispersions to thin films" J. Mat. Chem. C, 2017, 5, 12561 
26 V. K. LaMer and R. H. Dinegar "“Theory, Production and Mechanism of Formation of Monodispersed Hydrosols," J. Am. Chem. Soc., 1950, 17, 4847-4854

27 P. Ramanujam, B. Vaidhyanathan, J. G. P. Binner, S. Ghanizadeh, and C. Spacie "Solvothermal nanoYAG synthesis: Mechanism and particle growth kinetics" J. Supercrit. Fluids, 2016, 107, 433-440.

$28 \mathrm{M}$. Vorsthove, U. Kynast "Efficiency issues in Ce3+ doped YAG nanocrystals" Mater. Res. Bull., 2011, 46, 1761-1765

29 G. Dantelle, M. Salaün, R. Bruyère, S. Kodjikian, and A. Ibanez "Luminescent coatings prepared from optimized YAG:Ce nanoparticles" Thin Solid Films, 2017, 643, 36-42.

30 R. Kasuya, T. Isobe, H. Kuma, and J. Katano "Photoluminescence Enhancement of PEG-Modified YAG:Ce3+ Nanocrystal Phosphor Prepared by Glycothermal Method J. Phys. Chem. B, 2005, 109, 22126-22130.

31 A. Aboulaich, J. Deschamps, R. Deloncle, A. Potdevin, B. Devouard, G. Chadeyron, R. Mahiou "Rapid synthesis of Ce3+doped YAG nanoparticles by a solvothermal method using metal carbonates as precursors" New J. Chem., 2012, 36, 2493-2500

$32 \mathrm{~T}$. Isobe "Low-temperature wet chemical syntheses of nanocrystal phosphors with surface modification and their characterization" Phys. Status Solidi Appl. Mater. Sci., 2006, 203, 2686-2693

33 Y. Kamiyama, T. Hiroshima, T. Isobe, T. Koizuka, and S. Takashima "Photostability of YAG:Ce3+ Nanophosphors Synthesized by Glycothermal Method" J. Electrochem. Soc., 2010, 157, J149

34 M. Nyman, L. E. Shea-Rohwer, J. E. Martin, P. Provencio "Nano-YAG:Ce Mechanisms of Growth and EpoxyEncapsulation" Chem. Mater., 2009, 21, 1536-1542.

35 A. Revaux, G. Dantelle, N. George, R. Seshadri, T. Gacoin, J. P. Boilot "Improvement of luminescent properties and photostability of YAG nanoparticle-based films" Nanoscale, 2011, 3, 2015-2022.

36 G. Dantelle, D. Testemale, E. Homeyer, A. Cantarano, S. Kodjikian, C. Dujardin, J.L. Hazemann, A. Ibanez. "A new solvothermal method for the synthesis of size-controlled YAG:Ce single-nanocrystals" RSC Adv., 2018, 8, 26857-26870.

37 A. Herrmann, H. A. Othman, A. A. Assadi, M. Tiegel, S. Kuhn, C. Rüssel "Spectroscopic properties of cerium-doped aluminosilicate glasses" Opt. Mater. Express, 2015, 5, 720

38 L. Wang, L. Zhuang, H. Xin, Y. Huang, and D. Wang "Semiquantitative estimation of $\mathrm{Ce} 3+/ \mathrm{Ce} 4+$ ratio in YAG:Ce3+ phosphor under different sintering atmosphere" Open J. Inorg. Chem., 2015, 5, 12-18

39 A. D. Sontakke, J. Ueda, and S. Tanabe "Effect of synthesis conditions on $\mathrm{Ce} 3+$ luminescence in borate glasses" J. Non. Cryst. Solids, 2016, 431, 150-153

40 V.M. Orera, R.I. Merino, F. Pefia "Ce3+ $\leftrightarrow \mathrm{Ce} 4+$ conversion in ceria-doped zirconia single crystals induced by oxidoreduction treatments" Solid State lonics, 1994, 72, 224-231.

41 N. C. George, A.J. Pell, G. Dantelle, K. Page, A. Llobet, M. Balasubramanian, G. Pintacuda, B. F. Chmelka, R. Seshadri "The local environment of the activator ions in the solid state lighting Y3-xCexAl5O12" Chem. Mater., 2013, 25, 3979-3995.

42 P. Chaurand, W. Liu, D. Borschneck, C.Levard, M. Auffan, E. Paul, B. Collin, I. Kieffer, S. Lanone, J. Rose, J. Perrin "Multiscale X-ray computed tomography to detect and localize metal-based nanomaterials in lung tissues of in vivo exposed mice", Scientific reports, 2018, 8, 4408

43 M. Tella, M. Auffan, L. Brousset, E. Morel, O. Proux, C. Chanéac, B. Angeletti, C. Pailles, E. Artells, C. Santaella, J. Rose, A. Thiéry, J.-Y. Bottero "Chronic dosing of a simulated pond ecosystem in indoor aquatic mesocosms: fate and transport of CeO2 nanoparticles" Environ. Sci. Nano, 2015, 2, 653-663.
44 J.D. Cafun, K.O. Kvashnina, E. Casals, V.F. Puntes, P. Glatzel "Absence of $\mathrm{Ce} 3+$ Sites in Chemically Active Colloidal Ceria Nanoparticles" ACS Nano, 2013, 7, 10726-10732

45 S. E. Hunter, C. E. Ehrenberger, P. E. Savage "Kinetics and Mechanism of Tetrahydrofuran Synthesis via 1,4-Butanediol Dehydration in High-Temperature Water" J. Org. Chem., 2006, 71, 6229-6239

46 A. N. Bourns, Thesis "The vapour-phase dehydration of butanediols" McGill University, 1944.

$47 \mathrm{M}$. Inoue, T. Nishikawa, H. Kominami, T. Inui "Reactions of rare earth acetate hydrates in glycols at high temperatures" J. Mater. Sc. 2000, 35, $1541-1547$

$48 \mathrm{M}$. Inoue, H. Kominami, T. Inui "Thermal Reaction of Aluminum Alkoxide in Glycols" J. Am. Cer. Soc. 1990, 73, 11001102

49 P. Werle, "Alcohols, Polyhedral," Ullmann's Encyclopedia of Industrial Chemistry, Ed. Wiley-VCH, New York, 2011

50 E. V. Hort, P. Taylor, "Acetylene-Derived Chemicals," KirkOthmer Encyclopedia of Chemical Technology, Ed. Wiley-VCH, New York, 2001

51 S. Hosokawa, Y. Tanaka, S. Iwamoto, M. Inoue "Defect structure of rare-earth aluminum garnets obtained by glycothermal method", J. Alloys Compds, 2008, 451, 309-313

52 M.M. Xu, Z.J. Zhang, J.J. Zhu, J.T. Zhao, X.Y. Chen "Solvothermal Synthesis and Luminescence Properties of Yttrium Aluminum Garnet Monodispersed Crystallites with Well-Developed Faces" J. Phys. Chem. C 2014, 118, 27000-27009

53 D. Guo, B. Ma, L. Zhao, J. Qiu, W. Liu, Y. Sang, J. Claverie, H. Liu "Bright YAG:Ce Nanorod Phosphors Prepared via a Partial Wet Chemical Route and Biolabeling Applications" ACS Appl. Mater. 2016, 8, 11990-11997

54 N. T. K. Thanh, N. Maclean, S. Mahiddine "Mechanisms of Nucleation and Growth of Nanoparticles in Solution" Chem. Rev., 2014, 114, 7610-7630

55 D. Testemale, R. Argoud, O. Geaymond, J.-L. Hazemann “High pressure/high temperature cell for $\mathbf{x}$-ray absorption and scattering techniques" Rev. Scientific Instruments, 2005, 76, 043905

56 R. Battino, H.L. Clever "The solubility of gases in liquids" Chem. Rev. 1966, 66, 4, 395-463

57 http://www1.Isbu.ac.uk/water/nanobubble.html

$58 \mathrm{H}$. Kobayashi, S. Maeda, M. Kashiwa and T. Fujita, Measurements of ultrafine bubbles using different types of particle size measuring instruments, International Conf. Optical Particle Characterization (OPC 2014), ed. N. Aya, N. Iki, T. Shimura and T. Shirai, Proc. of SPIE 9232 (2014) 92320 U

59 D. E. Koppel "Analysis of Macromolecular Polydispersity in Intensity Correlation Spectroscopy: The Method of Cumulants" J. Chem. Phys. 1972, 57, 4814.

60 I. Flammer, J. Ricka "Dynamic light scattering with singlemode receivers: partial heterodyning regime" App. Opt. 1997, 36(30), 7508-7517.

61 Jared Lynch, Jiaqi Zhuang, Tie Wang, Derek LaMontagne, Huimeng Wu, and Y. Charles Cao "Gas-Bubble Effects on the Formation of Colloidal Iron Oxide Nanocrystals" J. Am. Chem. Soc. 2011, 133, 12664-12674

62 Naghmeh Fatemi, Zhengya Dong, Tom Van Gerven, and Simon Kuhn "Micro-bubbles as heterogeneous nucleation sites for crystallization in continuous microfluidic devices", Langmuir, 2019, 35, 60-69

63 A. Kordylla, T. Krawczyk, F. Tumakaka, G. Schembecker "Modeling ultrasound-induced nucleation during cooling crystallization", Chemical Engineering Science, 2009, 64 1635-1642

64 Z. Guo, A. G. Jones, H. Hao, B. Patel, and N. Li "Effect of ultrasound on the heterogeneous nucleation of $\mathrm{BaSO} 4$ during reactive crystallization" J. Applied Physics, 2007, 101, 054907 
65 A. Le Bail, Whole pattern decomposition methods and applications: a retrospection, Powder Diffr. 2012, 20, 316-326

66 O. Proux., E. Lahera, W. Del Net, I. Kieffer, M. Rovezzi, D. Testemale, M. Irar, S. Thomas, A. Aguilar-Tapia, E. F. Bazarkina, A. Prat, M. Tella, M. Auffan, J. Rose, J.-L. Hazemann "High-Energy Resolution Fluorescence Detected X-Ray Absorption Spectroscopy: A Powerful New Structural Tool in Environmental Biogeochemistry Sciences" J. Environ. Qual., 2017, 46, 1146-1157.

67 https://bruceravel.github.io/demeter/ 